\title{
The Frequency of Carbon-Enhanced Stars in HERES and SDSS
}

\section{Timothy C. Beers* ${ }^{\dagger}$}

Dept. of Physics \& Astronomy and JINA: Joint Institute for Nuclear Astrophysics, Michigan State University, E. Lansing, MI 48824 USA

E-mail: beers@pa.msu.edu

\section{Sara Lucatello}

Osservatorio Astronomico di Padova, Vicolo dell'Osservatorio 5, 35122, Padova, Italy

Email: lucatello@pd.astro.it

\section{Brian Marsteller, Thirupathi Sivarani}

Dept. of Physics \& Astronomy and JINA: Joint Institute for Nuclear Astrophysics, Michigan State University, E. Lansing, MI 48824 USA

E-mail: marsteller@pa.msu.edu, thirupathi@pa.msu.edu

\section{Paul Barklem, Norbert Christlieb}

Department of Astronomy and Space Physics, Uppsala University, Box 515, 751-20 Uppsala,

Sweden

Email: barklem@astro.uu.se, norbert@astro.uu.se

\section{Silvia Rossi}

Instituto de Astronomia, Geofísica e Ciências Atmosféricas, Departamento de Astronomia, Universidade de São Paulo, Rua do Matão 1226, 05508-900 São Paulo, Brazil

Email: rossi@astro.iag.usp.br

Recent large surveys of metal-poor stars in the Galaxy have revealed that a surprising fraction are enhanced in their carbon-to-iron ratios by factors of 10-10,000 relative to the solar ratio. Although the carbon overabundances in most of the stars in the metallicity interval $-2.7 \leq[\mathrm{Fe} / \mathrm{H}] \leq-2.0$ are likely to have arisen from Asymptotic Giant Branch processing (and subsequent dumping via mass transfer to a surviving companion), there exist many stars with $[\mathrm{Fe} / \mathrm{H}]<-3.0$ (including the two lowest $[\mathrm{Fe} / \mathrm{H}]$ stars known, with $[\mathrm{Fe} / \mathrm{H}]<-5.0)$ that may not be accounted for by this process. Rather, primordial (or nearly primordial) progenitors are implicated.

We report on the existing information from present surveys, including cool giants from the recently completed HERES (Hamburg/ESO R-process Enhanced Star) survey, and from warm main-sequence turnoff stars selected from SDSS-I. We also describe the results that will come from the recently-funded extension of the SDSS, which includes the program SEGUE: Sloan Extension for Galactic Understanding and Exploration. SEGUE will identify some 20,000 stars with $[\mathrm{Fe} / \mathrm{H}]<-2.0$, several thousand of which are expected to be carbon enhanced.

International Symposium on Nuclear Astrophysics - Nuclei in the Cosmos - IX

June 25-30 2006

CERN, Geneva, Switzerland 


\section{Introduction}

One of the most interesting results from modern surveys for metal-poor stars, such as the HK survey of Beers and colleagues [1, 2, 3] and the Hamburg/ESO Survey (HES) of Christlieb and collaborators [4], concerns the apparently large fraction of Carbon-Enhanced Metal-Poor (CEMP) stars identified at low metallicity. The fraction of CEMP stars among samples of stars with $[\mathrm{Fe} / \mathrm{H}]<$ -2.0 has been reported to be as high as $\sim 20-25 \%$ [5], which is significantly larger than the fraction observed at higher metallicities. Beers \& Christlieb (2005) [6] have pointed out that this fraction appears to increase with decreasing metallicity, reaching $\sim 40 \%$ for stars with $[\mathrm{Fe} / \mathrm{H}]<-3.5$. As discussed in [7] and [8], this increase in frequency may have an immediate consequence on the nature of the IMF in the early Galaxy, providing evidence for its being shifted toward higher masses with respect to the present-day IMF. Definitive evidence awaits additional study.

Given the impact of the fraction of CEMP stars at low metallicity on the understanding of early galactic chemical evolution, it is of crucial importance to assess it accurately. We make use of the data obtained by the Hamburg/ESO R-process Enhanced Star (HERES) survey [9, 10], which provides the presently largest sample of high-resolution spectra available for Very Metal-Poor stars (VMP; $[\mathrm{Fe} / \mathrm{H}] \leq-2.0$, according to the nomenclature of [6]). The late-type stars contained in HERES may suffer from dilution of their atmospheres due to convective mixing, which may alter the observed surface $[\mathrm{C} / \mathrm{Fe}]$, and hence the derived fraction of CEMP stars. Hence, we have also begun to look at the CEMP stars that are detected in the Sloan Digital Sky Survey (SDSS-I) [11]. These stars extend to generally higher effective temperatures than the HERES stars, and hence provide information that is not subject to alteration due to the evolution of the star.

\section{The HERES Data - Observations and Analysis}

It is important to note that the selection criteria for HERES stars was specifically set to minimize bias in the abundances of elements other than iron, including carbon, hence this sample is well-suited for the determination of the relative fractions of stars with various abundance signatures as a function of metallicity. Details of the observations and data reduction are discussed in [9] and [10]. Our analysis procedures are presented in [12].

\subsection{Chemical Abundance Measurements}

There are a total of 265 HERES stars with $[\mathrm{Fe} / \mathrm{H}] \leq-2.0$. Fifty four of these are CEMP stars, using the definition $[\mathrm{C} / \mathrm{Fe}] \geq+1.0$. The observed fraction of CEMP stars among VMP stars is thus $20 \pm 2 \%$. Figure 1 shows the measured $[\mathrm{C} / \mathrm{H}]$ and $[\mathrm{C} / \mathrm{Fe}]$ as a function of $[\mathrm{Fe} / \mathrm{H}]$ for the full HERES sample; solid lines indicate the adopted level of $\mathrm{C}$-enhancement for the stars that we consider CEMP stars. Notice that quite a number of stars lie only a small distance below this adopted cutoff.

The HERES sample covers a wide range of luminosities, extending from near the mainsequence turnoff up the red giant branch (RGB). It is expected that the depth of the stellar convective envelopes of these stars will vary considerably with evolutionary state, with the cool giants

\footnotetext{
* Speaker.

${ }^{\dagger}$ This work is supported, in part, by grants awarded by the US National Science Foundation
} 


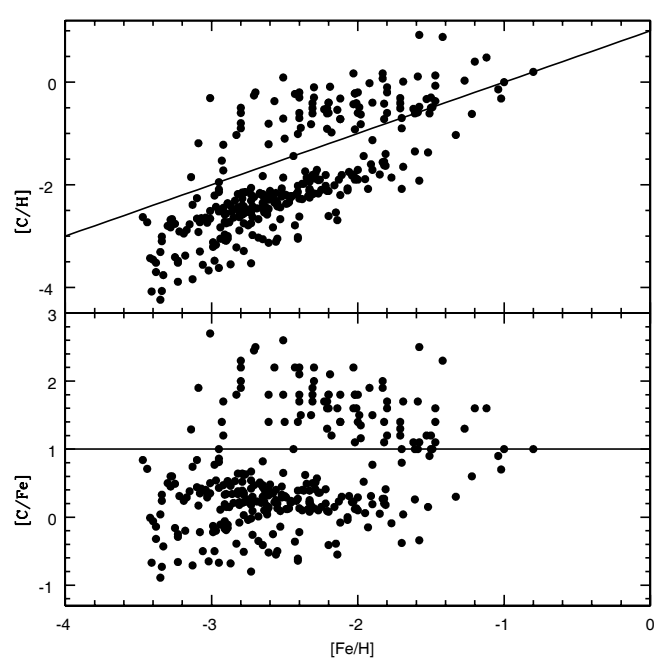

Figure 1: $[\mathrm{C} / \mathrm{H}]$ (top panel) and $[\mathrm{C} / \mathrm{Fe}]$ (lower panel) vs. $[\mathrm{Fe} / \mathrm{H}]$ for the total HERES sample. The lines indicate our original cutoff for considering a star to be a CEMP star, $[\mathrm{C} / \mathrm{Fe}]>+1.0$.

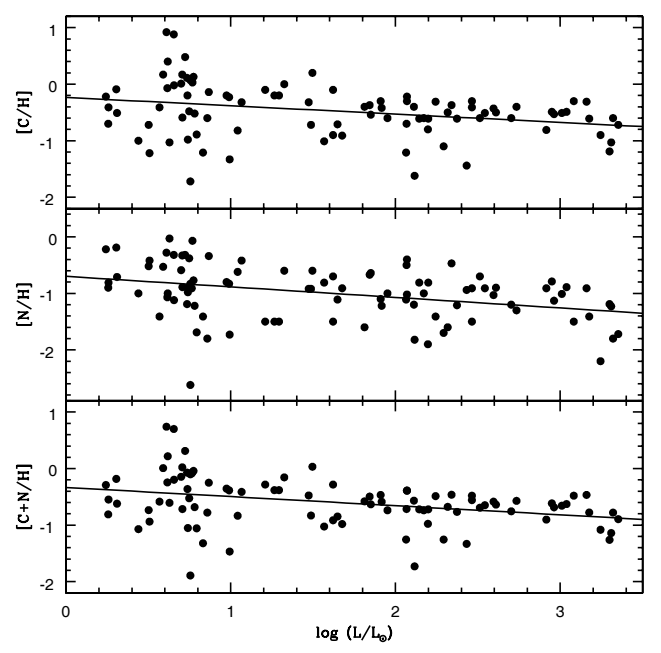

Figure 2: $[\mathrm{C} / \mathrm{H}]$ abundance (top panel), $[\mathrm{N} / \mathrm{H}]$ abundance (middle panel) and $[(\mathrm{C}+\mathrm{N}) / \mathrm{H}]$ abundance (bottom panel) as a function of luminosity for the HERES stars analyzed in the present paper. The solid lines are least-squares regression fits.

having the possibility of mixing a much larger amount of material than warmer dwarf and subgiant stars. Figure 2 shows the derived $\mathrm{C}, \mathrm{N}$, and $\mathrm{C}+\mathrm{N}$ abundances as a function of estimated $\log (\mathrm{L} / \mathrm{L} \odot)$. Inspection of this Figure reveals that the points are not distributed uniformly; there is a clear decreasing trend of $[\mathrm{C} / \mathrm{H}],[\mathrm{N} / \mathrm{H}]$, and $[(\mathrm{C}+\mathrm{N}) / \mathrm{H}]$ with luminosity. For the majority of these CEMP stars, which are expected to arise from AGB mass transfer from an evolved companion, we interpret this phenomenon as due to dilution of the transferred material with $\mathrm{H}$ from the receiving star, which progresses as the receiving star evolves up the RGB. The effect can be as much as 0.5 dex (or roughly a factor of three) over the range of luminosity we have observed. See [12] for additional details.

Due to the effects of evolutionary dilution, a number of stars that originally had $[\mathrm{C} / \mathrm{Fe}] \geq+1.0$ at their main-sequence stage will end up with a much lower $[\mathrm{C} / \mathrm{Fe}]$ value in their more advanced evolutionary stages, and would not qualify as CEMP stars according to our definition. Indeed, a CEMP fraction established on the basis of a sample that includes cool giants is, strictly speaking, always a lower limit. One way of resolving this problem would be to adopt a luminosity-dependent C-enhancement threshold. Aoki et al. (2006)[13] have proposed the following criterion: a star is considered a CEMP star if $[\mathrm{C} / \mathrm{Fe}] \geq+0.7$, for unevolved stars (up to $\log (\mathrm{L} / \mathrm{L} \odot)=2.3$ ), and $[\mathrm{C} / \mathrm{Fe}]$ $\geq+3-\log (\mathrm{L} / \mathrm{L} \odot)$ for stars of higher luminosity.

Figure 3 shows $[\mathrm{C} / \mathrm{Fe}]$ as a function of luminosity for the complete HERES sample. Also shown are two criteria for CEMP stars, $[\mathrm{C} / \mathrm{Fe}] \geq+1.0$ for all evolutionary phases, and the Aoki et al. (2006) luminosity-dependent criterion. If we adopt the Aoki et al. definition, a greater number of CEMP stars are included, and the resulting fraction of CEMP stars in the HERES sample rises to $23 \pm 2 \%$. The Aoki et al. criterion does not adequately account for possible alterations in the surface carbon abundances at luminosities below $\log \left(\mathrm{L} / \mathrm{L}_{\odot}\right)=2.3$, where, starting at luminosities as low 


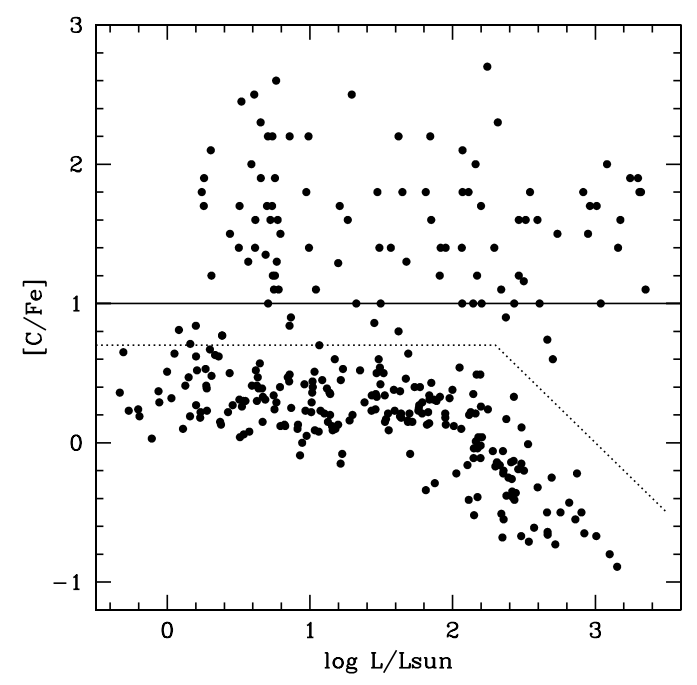

Figure 3: $[\mathrm{C} / \mathrm{Fe}]$ as a function of luminosity for the full HERES sample. The solid line represents the cutoff for CEMP stars adopted in the present paper, the dotted line is that proposed by [13], calculated by assuming a mass of $0.8 \mathrm{M}_{\odot}$ for all stars in the sample.

as $\log \left(\mathrm{L} / \mathrm{L}_{\odot}\right)=0.8$, the first dredge-up occurs and the convective envelope mass starts increasing. Therefore, such a criterion, although a step in the right direction, does not fully take into account the effects of evolutionary dilution.

\section{The SDSS Data - Observations and Analysis}

Because of the potentially large effects on the calculated fraction of CEMP stars due to evolutionary dilution, it is clearly advantageous to obtain large samples of non-evolved stars, from which the frequency of carbon-enhanced stars can be calculated without this additional concern. Fortunately, just such a sample is available, based on medium-resolution $(R=2000)$ spectroscopy of metal-poor candidates chosen as calibration objects in the SDSS. This data is now publicly available for the full SDSS-I data set (DR-5).

We have developed sets of procedures by which one can estimate $[\mathrm{C} / \mathrm{Fe}]$ and $[\mathrm{Fe} / \mathrm{H}]$ for these data based on either application of the calibrations described by [14] or, a newly developed semiautomated technique based on spectral synthesis of the CaII $\mathrm{K}$ and $\mathrm{CaII} \mathrm{H}$ lines, along with the $\mathrm{CH}$ G-band at $4300 \AA$, which will be reported on in due course (Marsteller et al., in preparation).

The results to date appear quite encouraging. We have been able to obtain measurements of $[\mathrm{Fe} / \mathrm{H}]$ and measurements (or upper limits) on $[\mathrm{C} / \mathrm{Fe}]$ for the over 5000 metal-poor main-sequence turnoff stars contained in SDSS-I. Once these results have been validated with calibration observations taken at high spectral resolution, we will be able to confidently assess the frequency of CEMP stars based on a sample that should be unaffected by the evolutionary effects noted above.

Furthermore, based on tests conducted to date, the Sloan Extension for Galactic Exploration and Understanding (SEGUE) project within SDSS-II will yield identifications of over 20000 stars with $[\mathrm{Fe} / \mathrm{H}]<-2.0$, including stars across a wide range of evolutionary stages. This sample 
will make it feasible to construct empirical measures of the dependence of C-enhancement on evolutionary state, and thereby allow one to correct for it in the future.

\section{References}

[1] Beers, T. C., Preston, G. W., \& Shectman, S. A. 1985, A Search for Stars of Very Low Metal Abundance, AJ, 90, 2089

[2] Beers, T. C., Preston, G. W., \& Shectman, S. A. 1992, A Search for Stars of Very Low Metal Abundance. II. , AJ, 103, 1987

[3] Beers, T. C., Low-Metallicity and Field Horizontal-Branch Stars in the Halo of the Galaxy, in Third Stromlo Symposium: The Galactic Halo, eds. B. K. Gibson, T. S. Axelrod, \& M. E. Putman, ASP Conference Series, 165, p. 202

[4] Christlieb, N. 2003, Finding the Most Metal-Poor Stars of the Galactic Halo with the Hamburg/ESO Objective-prism Survey, Rev. Mod. Astron, 16, 191

[5] Marsteller, B., Beers, T. C., Rossi, S., Christlieb, N., Bessell, M., \& Rhee, J. 2005, Carbon-Enhanced Metal-Poor Stars in the Early Galaxy, Nuclear Physics A, 758, 312

[6] Beers, T. C., \& Christlieb, N. 2005, The Discovery and Analysis of Very Metal-Poor Stars in the Galaxy, ARA\&A, 43, 531

[7] Abia, C., Dominguez, I., Straniero, O., Limongi, M, Chieffi, A., \& Isern, J. 2001, The Implications of the New Z=0 Stellar Models and Yields on the Early Metal Pollution of the Intergalactic Medium, ApJ, 557, 126

[8] Lucatello, S., Gratton, R. G., Beers, T. C., \& Carretta, E. 2005, Observational Evidence for a Different IMF in the Early Galaxy, ApJ, 625, 833

[9] Christlieb, N., et al. 2004, Hamburg/ESO R-process Enhanced Star Survey (HERES). I. Project Description, and Discovery of Two Stars with Stron Enhancements of Neutron-Capture Elements, $A \& A, \mathbf{4 2 8}, 1027$

[10] Barklem, P. S., Christlieb, N., Beers, T. C., Hill, V., Holmberg, J., Marsteller, B., Rossi, S., \& Zickgraf, F.-J. 2005, Hamburg/ESO R-process Enhanced Star Survey (HERES). II. Spectroscopic Analysis of the Survey Sample, A\&A, 439, 129

[11] York, D. et al. 2000, The Sloan Digital Sky Survey: Technical Summary, AJ, 120, 1579

[12] Lucatello, S., Beers, T. C., Christlieb, N., Barklem, P. S., Rossi, S., Marsteller, B., Sivarani, T., \& Lee, Y. 2006, The Frequency of Carbon-Enhanced Metal-Poor Stars in the Galaxy from the HERES Sample, ApJL, submitted

[13] Aoki, W., Beers, T. C., Christlieb, N., Norris, J. E., Ryan, S. G., \& Tsangarides, S. 2006, Carbon-Enhanced Metal-Poor Stars. I. Chemical Compositions of 26 Stars, ApJ, submitted

[14] Rossi, S., Beers, T. C., Sneden, C., Sevastyanenko, T., Rhee, J., \& Marsteller, B. 2005, Estimation of Carbon Abundances in Metal-Poor Stars. I. Application to the 'Strong G-Band' Stars of Beers, Preston, \& Shectman, AJ, 130, 2804 